\title{
GESNERUS
}

Vierteljahrsschrift für Geschichte der Medizin und der Naturwissenschaften

Revue trimestrielle d'histoire de la médecine

Jahrgang - Volume - $21945 \quad$ Heft - Fasc. - 1

\section{Conrad Geßner als Balneologe}

Daß Conrad Geßner der früheste Naturforscher der Schweiz war, ist allgemein bekannt; weniger bekannt dürfte es sein, daß er mit Paracelsus zu ihren ersten Balneologen gehörte. Er hat als erster in größerem Umfang schweizerische Heilquellen untersucht, nach ihrer Zusammensetzung - soweit das möglich war — wie auch nach ihrer therapeutischen Wirkung. Geßner hat selber Bade- und Trinkkuren gemacht und, wie es seine Gewohnheit war, den therapeutischen Einfluß dieser Heilmittel an sich selber nachgeprüft. Es soll damit freilich nicht gesagt sein, er sei überhaupt der erste schweizerische Bäderschriftsteller und Balneologe gewesen. An «Salzbrunnen» hatten die Regierungen aus naheliegenden Gründen frühzeitig Interesse. So ließ die Berner Regierung im Jahr 1480 in Riggisberg, einem Dorf an der Straße von Bern nach Gurnigel, von ihrem Stadtarzt Meister Peter, einem ehemaligen Bar. füßer, eine angebliche Salzader beschwören, die man zuvor durch Graben nicht hatte finden können. Meister Peter machte sich «mit der Tüflen lügenhaften Kunst», wie der Berner Chronist Valerius Anshelm berichtet, «hinter das Geschäft, als ob der Fiend alles menschlichen Guts dem Menschen mehr zu Gut solte vermögen und thun, dann Gott, ihrer beider Herr, des Tüfels zorniger Verdammer und des Menschen gütiger Be. gnader». ${ }^{1}$ Die Beschwörung hatte anscheinend keinen Erfolg. Über die Bäder von Baden schrieb 1489 der Frühhumanist Heinrich Gundelfingen eine nie veröffentlichte, Conrad Geßner aber zur Einsicht vorgelegene Schrift «De Thermis Badensibus».2 Über dasselbe Bad schrieb 1516

1 Über Valerius Anshelm vergl. «Große Schweizer Forscher». Zürich 1939, S. 25 f., mit Lit.-Angaben. Die Berner Chronik des V. A. wurde hgg. v. hist. Verein Bern, 1884; über Meister Peter s. Bd. 1, S. 162 f.

${ }^{2}$ Heinrich Gundelfingen, ca. 1440-1490, von Konstanz gebürtig, Kaplan in Freiburg 
Alexander Sytz, (1470-1540), Arzt, ein oft vertriebener, oft heimatlos wandernder religiöser Sucher und wohl auch Eiferer, der sich am Ende seines Lebens den Wiedertäufern anschloß, eine kleine Arbeit, «Menschlich Lebens Art und Ursprung und wie man das befristen soll durch die Wildbäder bevor zu Oberbaden», die in Basel im Druck herauskam. ${ }^{3}$ Über Pfäffers hatte schon in der ersten Hälfte des 15. Jhds. Dr. Felix Hämmerlin, der berühmte Zürcher Chorherr, (1388-1458), eine Geßner ebenfalls bekannte Schrift verfaßt. ${ }^{4}$ Im Jahr 1500 beschrieb der Zürcher Stadtarzt Dr. Volmar das äußere Gyrenbad im Zürcher Tößtal, in welchem er Alaun mit Vitriol, Kupfer und Schwefel nachgewiesen haben wollte und das er für eine große Reihe von Krankheiten empfahl. ${ }^{5}$ Sicher sind damals auch noch andere Badeschriften entstanden, die uns heute nicht mehr bekannt sind. Die ersten bedeutenden Bäderschriften waren aber zweifellos diejenigen von Theophrastus Paracelsus (1493-1541) über St. Moritz und Pfäffers aus dem Jahre $1535 .{ }^{6}$

Gleichwohl war Geßner der erste, der sich in ausgedehntem Maß mit einer Untersuchung der Schweizer Heilquellen befaßte. Solche Untersuchungen lagen gerade damals im Zug der Zeit, wobei die wissenschaftliche Balneologie von Italien ausging. Viele der einschlägigen Schriften wurden in Venedig gedruckt. Von Zeitgenossen Geßners seien nur die beiden berühmten italienischen Ärzte Falloppio und Gratarolus genannt. Gabriel Falloppio aus Modena (1523-1562), ${ }^{7}$ bekannt durch seine anatomischen und chirurgischen Schriften, wurde 1548 Medizinprofessor erst in Ferrara und dann in Pisa, um 1551 eine Professur für Anatomie

i. Br., später Chorherr in Bern und Prof. f. Rhetorik in Freiburg i. B., von Geßner irrtümlich Gundelfinger genannt. Bekannt durch seinen Bericht über Niklaus von der Flüh (1488) und histor. Arbeiten. Über ihn Zedler, Universallexikon, Halle und Leipzig, Bd. 11, S. 1394, (1735) und Hist. biogr. Lexikon d. Schweiz, Bd. 4, S. 16, mit Lit.-Angaben.

3 Alexander Sytz oder Seitz. Über ihn vergl. Biogr. Lexikon d. hervorr. Ärzte, (1934), hgg. v. Haberling-Hübotter-Vierordt, Bd. 5, S. 125, mit Lit.-Angaben. Er schrieb 1509 ein Traktätlein über die Syphilis und 1516 ein merkwürdiges Büchlein über Träume.

4 Felix Hämmerlin oder Hemmerlin: Vergl. Allg. D. Biogr., Bd.11, S. 721-724, und H. Walser, Meister Hemmerli und seine Zeit, Zürich, 1940. Die v. Geßner zitierte Schrift «De balneis naturalibus» wird sonst nirgends zitiert und scheint verloren zu sein.

5 Über die Badeschrift von Dr. Volmar vergl. Gsell-Fels, Bäder und Kurorte der Schweiz, 2. Aufl., Zürich 1890, S. 527. Die Schrift selber habe ich im Original nicht auffinden können.

${ }^{6}$ In der Gesamtausgabe von Sudhoff (1925) Bd. 9, S. 639 ff. über Pfäffers.

7 Über Falloppio vergl. med.-hist. Handbücher; ferner das «Lexikon hervorr. Ärzte», wie auch die «Enciclopaedia Italiana» Bd. 14, S. 755 (1932), mit Bild. 
und Botanik in Padua zu übernehmen, die er bis zu seinem frühen Tod innehatte. Posthum kam eine 1564 in Venedig erschienene Schrift heraus «Gabrielis Falloppii Mutinensis medici ac philosophi praestantissimi de medicatis Aquis et de Fossilibus». Im 9. Kapitel dieses Buches gab er genaue Anweisungen, auf welche Weise das Wasser von Bädern und Heilquellen auf ihre qualitative Zusammensetzung zu untersuchen sei; Seite $30 \mathrm{a}-37 \mathrm{~b}$ handelt "De ratione et modo investigandi et cognoscendi quid unaquaeque thermalis aqua habet in se commixtum». Diese Anweisungen hat Geßner hoch geschätzt und später in seinen «köstlichen Arzneischat» des Euonymus Philiater ${ }^{8}$ aufgenommen, dessen zweiter Teil, der diese Anweisungen enthält, freilich erst nach seinem Tod herauskam, als «Conradi Gesneri Medici et Philosophi Tigurini Evonymus, sive de Remediis secretis, pars secunda: nunc primum opera et studio C. Wolphio, Medici, Physici Tigurini in lucem editus», ohne Druckortangabe und ohne Jahrzahl, aber mit einem 1569 datierten Vorwort Wolfs. Auch Falloppio kannte Geßners balneologische Arbeit über die Heilquellen Helvetiens und Germaniens, die 1553 im Sammelwerk «Corpus Venetum de Balneis» bei Juntas in Venedig erschienen war. Wo er in seiner Arbeit auf den Bleinachweis zu sprechen kommt, der ihm selber nie gelungen sei, bezieht er sich auf Conrad Geßner (S. 25 seines Werks). Dieser hatte den Bleinachweis freilich nur etymologisch geliefert, indem er annahm, daß das Vogesenbad Plombières und das belgische Bad Plummers den Namen vom Bleigehalt her trügen. Falloppio beschrieb nur italienische Bäder, die er allein aus eigener Anschauung kannte, da er sein Heimatland offenbar nie verlassen hat. Anders Guilelmus Gratarolus. Auch er gehörte zu den berühmtesten italienischen Ärzten seiner Zeit. Wie Geßner im

8 Conradi Gesneri Medici et Philosophi Tigurini Euonymus, sive de Remediis secretis pars secunda: nunc primum opera et studio Caspari VVolphii Medici, Physici Tigurini in lucem editus, Tiguri. - Modus destillandi aquam simplicem et aquas fontium thermalium ut in cognitionem rerum his admixtarum et inde facultatum earundem deveniamus, ex Gabrielis Falloppij libro de aquis medicatis, pg. $24 \mathrm{ff}$.

Köstlicher Arztneyschatz deß wolerfarenen und weytberümpten Euonymi Philiatri etc. Des köst. Artzneyschatzes Evonymi Philiatri ander theil - verteutschet durch Joh. Jak. Nüscheler, der Artzney D. zu Zürych. Getruckt zu Zürych im Jar 1608, S. 32 ff. In der deutschen Fassung läßt Nüscheler Falloppio Kupfer mit Galläpfelsaft nachweisen, was auf einem Übersetzungsfehler beruht. Calcanthum (S.26 im lat. Original) ist ein Gemisch, das hauptsächlich Eisenvitriol enthält. Man vergl. darüber Frieboes, Aulus Cornelius Celsus, 2. Aufl., Braunschweig, 1906, S. 675/6 mit Lit.-Angaben. Die Übersetzung der meisten Wörterbücher ist falsch. 
Jahre 1516 geboren, in Bergamo, starb er drei Jahre nach diesem, in Basel. Er hatte in Padua Philosophie und Medizin studiert und hielt von 1537-1539 Vorlesungen über Avicenna, um sich nachher in seiner Geburtsstadt als Arzt niederzulassen. Er machte weite Reisen durch Italien, Savoyen, Burgund und die Schweiz, und ließ sich, Protestant geworden, schließlich in Basel nieder. Einige Zeit bekleidete er eine Medizinprofessur in Marburg, um dann für den Rest seiner Lebenszeit wieder nach Basel zurück zu kehren. ${ }^{9}$ Mit Gratarolus verbanden Geßner persönliche Beziehungen. Er erhielt von ihm z. B. eine Beschreibung des bündnerischen Heilbads Le Prese im Puschlav. Gratarolus war auch Mitarbeiter am «Corpus Venetum de Balneis» mit einem Beitrag «De Thermis Rheticis agri Bergomatis». Er hat auch die Quellen von St. Moritz und Alvaneu untersucht und geschildert und zusammen mit Geßner und Mattioli die berühmten Bäder von Bormio. Pierandrea Mattioli (1517-1577) war lange Zeit Leibarzt am Hof Ferdinands I. und berühmt als Arzt und Botaniker. Sein Kräuterbuch, in verschiedene Sprachen übersetzt, ist in über sechzig Ausgaben erschienen. ${ }^{10} \mathrm{Da} ß$ sich die schweizerischen Quellen schon im 16. Jhd. über die Landesgrenzen hinaus eines guten Rufes erfreuten, ersieht man daraus, daß sich schon damals wie in den folgenden Jahrzehnten auch ausländische Ärzte mit ihnen befaßten, wie der Italiener Andrea Baccius von Mailand (gest. 1600), Verfasser eines bedeutenden und umfangreichen Werkes über Bäder, ${ }^{11}$ oder Leonhard Fuchs in Tübingen (1501-1566), aus dessen medizinischem Kompendium «De curandi ratione libri octo», 1548 im Druck erschienen, auch Exzerpte über Bäder und Badwesen ins Corpus Venetum aufgenommen wurden und der Geßner ziemlich oft als Gewährsmann diente. ${ }^{12}$

9 Über Gratarolus vergl. u. a. A. Burckhardt, Gesch. d. med. Fakultät z. Basel, Basel, 1907, S. 53, wo er indessen doch wohl etwas unterschätzt wird.

10 Mattioli, vergl. «Lexikon hervorr. Ärzte», Bd. 4, S. 119 mit reicher Lit.-Angabe Moebius, Gesch. d. Botanik, Jena, 1937, S. 33 und C. Meyer-Ahrens. Die Heilquellen d. Schweiz, 2. Aufl. 1867, S. 574. Gleich M. A. konnten auch wir das angebl. 1540 erschienene Werklein über die Bäder von Bormio nicht finden.

11 Andrea Baccius. Vergl. «Lexikon hervorr. Ärzte», Bd. 1, S. 270 und med.-hist. Handbücher. Sein noch heute interessantes Bäderwerk trägt den Titel «De thermis, lacubus, fluminibus, balneis totius orbis». Es erschien 1571 erstmals in Venedig und bis 1711 noch drei weitere Male.

12 Leonhard Fuchs. Vergl. «Lexikon hervorr. Ärzte», Bd. 2, S. 637-639 und medizinhist. Hand- und Lehrbücher. Gleich Geßner auch berühmter Botaniker. Vergl. Möbius, S. 27, l. c. Als Balneologe unbekannt, von Geßner aber oft zitiert. 
Unter seinen schweizerischen Kollegen fand Geßner nicht manchen, der sich als Badeschriftsteller betätigt hätte. In Sitten lebte der 1520 geborene Arzt und Apotheker Kaspar Ambühl, Collinus genannt, ein bedeutender Gelehrter und Freund Geßners, der ein Werk über die Heilquellen des Wallis geschrieben hat. ${ }^{13}$ Dr. Joachim von Watt, der berühmte St. Galler Stadtarzt und Bürgermeister (1483-1551) war ein warmer Freund diätetisch-physikalischer Therapie. ${ }^{14} \mathrm{Ob}$ er aber wirklich eine Schrift über das Rietbad im Toggenburg herausgegeben hat, wie in der alten Bäderliteratur und selbst bei Meyer-Ahrens zu lesen ist, ${ }^{15}$ erscheint doch recht fraglich. ${ }^{16}$

In Zürich begründete Geßner geradezu eine balneologische Tradition, nicht von Badeärzten, wohl aber von Bäderschriftstellern. Keine andere Schweizerstadt hat eine gleich stattliche Menge balneologischer Schriftsteller hervorgebracht. Erwähnt seien nur J. Ziegler (1591-1670), Heinrich Lavater (1611-1691), J. J. Wagner (1641-1695), Joh. v. Muralt (1645-1733), Salomon Hottinger (1649-1713), J.J.Scheuchzer (16721733), mit Ausnahme von Hottinger alle auch Nachfolger Geßners im Amt eines Stadtarztes und teilweise gleich ihm bedeutende Naturforscher. ${ }^{17}$ Im 19. Jahrhundert war Konrad Meyer-Ahrens (1813-1872)

13 Caspar Ambühl, Collinus genannt. Vergl. hist. biogr. Lexikon d. Schweiz, Bd. 1, S. 336 und Meyer-Ahrens, 1. c., S. 145 u. a. O. Er verfaßte 1574 ein Werklein über die Heilquellen der Seduner, das ich indessen nirgends finden konnte.

14 Joachim von Watt, Vadian genannt. Über diesen bedeutendsten Humanisten der Schweiz erscheint in diesen Tagen der erste Band der groß angelegten Biographie des in Bern tätigen St. Galler Historikers W. Näf. Über Vadian als Arzt vergl. B. Milt, Ein Konsilium Vadians, Schw. Med. W., 62. Jhrgg., 1932, S. 260 ff.

15 Druckschrift über das Rietbad aus der St. Galler Klosterdruckerei vom J. 1677, die noch mehrnals aufgelegt wurde; Meyer-Ahrens 1. c. S. 425. Die Beschreibung des Bades durch Vadian wurde abgedruckt in der NZZ, 1944, Nr. 934 in «Altes und Neues vom Rietbad» von J. Wickle.

$16 \mathrm{Da} \beta$ die Beschreibung in d. vorl. Form nicht von Vadian stammt, dürfte für keinen Vadiankenner fraglich sein, sprachlich nicht und inhaltlich nicht. Wahrscheinlich ist sie erheblich später entstanden und sollte Vadians Name propagandistische Wirkung tun. Daß er das Bad kannte, ist außer Frage. Hätte er je eine Schrift darüber verfaßt, wenn auch in anderer Form, wäre dies Geßner, der das Bad in seiner Bäderschrift erwähnt, kaum verborgen geblieben, der eine solche Schrift aber nirgends erwähnt, weder in seiner Bibliotheca im Artikel über Vadian, noch in seinem Bäderbuch bei Erwähnung des betr. Bades. Wahrscheinlich hat nie eine solche bestanden.

17 Vergl. über diese Männer R. Wolf, Biogr. z. Kulturgeschichte d. Schweịz, Zürich, 1858-1862, ferner Neujahrsbl. z. Besten des Waisenhauses Nr. 93 (1871), Die Ärzte Zürichs. Thre balneolog. Arbeiten findet man bei Meyer-Ahrens l. c. angeführt, 
gleich bedeutend als Balneologe wie als Medizinhistoriker und zugleich der Historiker der schweizerischen Bäderkunde. ${ }^{18}$ Seinem Werk über «Die Heilquellen und Kurorte der Schweiz», erstmals 1860 in Zürich herausgekommen, ist die vorliegende Arbeit in mancher Hinsicht verpflichtet. Auch die Medizinprofessoren der jungen Zürcher Hochschule stellten sich eifrig in den Dienst der Erforschung schweizerischer Heilquellen, allen voran ihr erster Inhaber des Lehrstuhls für innere Medizin, J. Lucas Schönlein (1793-1865), der nicht nur den Ruf des Wildegger Jodwassers zu medizinischem Gebrauch begründete, sondern erneut auf die heilenden Kräfte der Quellen von Schuls-Tarasp aufmerksam machte, die beinahe in Vergessenheit geraten und nur mehr von den Einheimischen benutzt worden waren. ${ }^{19}$ Sein Nachfolger auf dem Zürcher Lehrstuhl, Karl von Pfeufer (1806-1869), machte als erster auf den medizinischen Gebrauch des Birmensdorfer Schwefelwassers aufmerksam, dessen Versand hauptsächlich ihm seinen bedeutenden Aufschwung verdankte. ${ }^{20}$

Nach Conrad Geßners Tod wurde freilich sein geistiges Erbe, seine vornehmlich beschreibende, eklektische Methode in Naturwissenschaft und Medizin, zunächst nicht in Zürich, sondern in Basel, von Baslern weiter gepflegt. Wohl war sein Schüler und Amtsnachfolger, der nicht unbedeutende Zürcher Caspar Wolf (1532-1601) als Geßners literarischer Testamentsvollstrecker in sehr verdienstlicher Weise tätig, ohne dabei aber aus seiner Famulusrolle hinauszuwachsen. Die wirklichen geistigen Erben waren die Basler Felix Plater (1536-1614), Stadtarzt und Medizinprofessor in der Rheinstadt und Johannes Bauhin (1541-1613), «Lybmedicus, Consiliarius und geheimer Rath» des Grafen Ulrich von Württemberg-Mömpelgard. Plater übernahm nach Geßners Tod einen Teil seiner Sammlungen; die Originalbilder zu Geßners Vogelbuch befinden sich aus seinem Besitz noch heute in der Universitätsbibliothek

18 Über diesen bedeutenden Schweizer Medizinhistoriker und Balneologen s. «Lexikon d. hervorr. Ärzte», Bd. 4, S. 189 und «Corresp. Blatt f. Schw. Ärzte», 1873, S. 121. Seine «Heilquellen der Schweizs sind eine Fundgrube für medizinhist. Material; leider ist aber die Quellenangabe oft sehr dürftig und das Material nicht immer aus erster Hand.

19 Über Schönlein vergl. «Lexikon d. hervorr. Ärzte», Bd. 5, S. 123 mit guten Lit.-Angaben; über seine Zürcher Jahre noch O. Nägeli, Die med. Auffassungen v. J. L. Sch. vor 1.00 Jahren, Schw. Med. W., 1933, Nr. 17, S. 398 ff. und B. Milt, Eine Krise d. abendl. Heilkunde und ihre Überwindung, Vierteljahrsschr. d. Zürch. natf. Ges., Bd. 87, 1942, S. 507 ff. Sch. und die Wildegger Jodquelle s. Meyer-Ahrens 1. c., S. 726.

${ }_{20}$ Karl v. Pfeufer, vgl. Allg. D. Biogr., Bd. 25, S. 661 und Meyer-Ahrens 1. c., S. 723, 21 C. Wolf, vergl. R. Wolf, 1. c. Bd. 1, S. 43-56. 
Basel. $^{22}$ Bauhin, ein direkter Schüler Geßners wie auch von Leonhard Fuchs, hat wie kein anderer Geßners botanische Studien fortgeführt; von seiner Hand finden sich auch in Geßners hinterlassenem Pflanzenwerk verschiedene Notizen. Er wäre voraussichtlich der einzige gewesen, der nach des Meisters vorzeitigem Tod dieses Werk hätte herausgenen können. Bauhin hat Geßner im Jahr 1561 auf einer beinahe einen Monat dauernden Reise ins Bündnerland begleitet, welche nicht zu botanischen Zwecken allein, sondern ebenso aus balneologischen Gründen unternommen worden war. ${ }^{23}$ Bauhin hat später ebenfalls eine umfangreiche Bäderschrift verfaßt «Joanni Bauhini Historia fontis et balnei Bollensis» die in Mömpelgard gedruckt herauskam und 1603 in deutscher Sprache auch in Stuttgart aufgelegt wurde, als «Johanni Bauhini Arznei und Badbuch». Wichtiger als diese Veröffentlichung war die Bäderschrift eines andern Basler Arztes aus dieser Epoche, welche die Bäder von Baden nach langjähriger Erfahrung ihres Badearztes schilderte; sie stammte aus der Feder des Medizinprofessors Dr. Heinrich Pantaleon (1522-1599), der sich auch durch biographische Publikationen einen Namen gemacht hat. ${ }^{24}$ Seine Arbeit ist sehr lebendig geschrieben und beruft sich auf fünundzwanzigjährige Erfahrung an vielen Patienten und an sich selbst. Ein artiger Zufall wollte es, daß auch die balneologischen Bemühungen von Paracelsus von einem Basler Arzt weitergeführt wurden, dem Alchimisten Leonhard Thurneißer (1531-1596), einem viel geschmähten und doch immer wieder neu interessierenden Mann. ${ }^{25}$ Er gab im Jahr 1572 ein umfangreiches Bäderwerk heraus, das den Titel trug «Pison, 10 Bücher von kalten und warmen, minerischen und mettalischen Wassern", in Frankfurt a. d. O. erschienen ist und nach seinem Tod, im Jahr 1602, in Straßburg neu aufgelegt wurde. Wenn allerdings der Chemiehistoriker Kopp behauptete, Thurneißer habe in diesem Buch als erster die Methode empfohlen, die Mineralwasser durch Abdampfen und Prüfung des Rück-

22 F. Plater, a. gl. O., Bd. 4, S. 1-24 und neuere Spezialliteratur von Karcher, Rytz u. a.

23 J. Bauhin, a. gl. O., Bd. 3, S. $68 \mathrm{ff}$.

24 H. Pantaleon, vgl. Burckhardt, l. c., S. 49 ff. Seine Bewertung ist völlig ungerecht. Nach Meyer-Ahrens ist sein, Burckhardt übrigens unbekanntes, Werk über Baden die bedeutendste schweiz. Bäderschrift, die wir aus frühern Jahrhunderten aus der Schweiz besitzen. Sie erschien 1578 in Basel und trägt den Titel «Warhafftige und fleißige beschreibung der Uralten Stadt und Graveschafft Baden, sampt jhrer heilsamen warmen Wildbedern». Eine liebevollere Darstellung findet man in Zedlers Universallexikon.

25 Über Thurneyßer vgl. vor allem Kopp, Die Alchemie in älterer und neuerer Zeit, Heidelberg, 1886, Bd. 1, S. 107 ff.; ferner Kopp, Gesch. d. Chemie. 
standes zu untersuchen, irrte er sich. ${ }^{26}$ Diese Methode wurde lange vorher schon von Conrad Geßner angewandt und vor allem von Falloppio sorgfältig ausgebaut in der oben erwähnten Schrift, welche auch Geßner am Ende seines Lebens noch zu Rate ziehen konnte.

Der Wert aller dieser Bäderschriften ist heute ein rein historisch-beschreibender. Die brauchbaren chemischen Analysen stammen wohl ausschließlich aus dem 19. Jahrhundert. Merkwürdigerweise steht es mit den klinisch-therapeutischen Angaben nicht viel besser. Das ist vielleicht weniger erstaunlich, wenn man weiß, daß sich doch die meisten dieser Schriftsteller nur auf anderer Leute Erzählungen verlassen mußten und keine oder nur wenig eigene Erfahrung als praktisch tätige Badeärzte besaßen. So waren diese von Medizinern verfaßten Bäderschriften nicht viel wertvoller als etwa diejenigen der zeitgenössischen Geographen, welche diese Bäder ebenfalls kurz beschrieben, wie etwa Sebastian Münster von Basel (1489-1552), auf dessen "Cosmographia», 1544 zum ersten Mal gedruckt, sich auch Geßner verschiedentlich bezog, ${ }^{27}$ Johannes Stumpf $(1500-1566)$ in seiner 1547 erschienenen Schweizerchronik ${ }^{28}$ und Egidius Tschudi (1505-1572) von Glarus, der Vater der Schweizergeschichte, auf dessen Schrift «Liber de alpinis gentibus» von 1538 sich Geßner in seiner eigenen Bäderschrift verschiedene Male berief. ${ }^{29}$

Um nun aber wieder zu Conrad Geßner selber zurückzukehren, soll zunächst von seinem im mehrfach erwähnten "Corpus Venetum de Balneis» veröffentlichten Bäderwerk die Rede sein. ${ }^{30}$ Dieses 1553 bei Juntas in Venedig herausgekommene umfangreiche Werk war die erste große Sammlung von Bäderschriften, welche das wesentliche Schrifttum über die Bäder von Hippocrates' Zeit über die römische, alexandrinische, arabische und mittelalterliche bis auf die eigene Zeit umfaßte, teilweise in Form von Auszügen aus den betreffenden Schriftstellern, teilweise als selbständige

${ }^{26}$ Kopp, Gesch. d. Chemie; von ihm übernahm diese Angabe R. Wolf, 1. c. Bd. 3, S. 33.

27 S. Münster vgl. R. Wolf, 1. c. Bd. 2, S. 1-26.

28 J. Stumpf, vgl. J. C. Gasser, Denkschr. auf St., in ZSG. 1931.

${ }_{29}$ E. Tschudi, vgl. HBL, Bd. 7, S. 79 f., mit Lit.-Angaben. Über diese Männer als Geographen versch. Artikel von Leo Weiß, Zürich, von dem noch eine zusammenfassende Würdigung ihrer Leistungen zu erwarten ist.

30 De Balneis, omnia quae extant apud Graecos, Latinos et Arabas ... In quo Aquarum et Thermarum omnium quae in toto fere orbe terrarum sunt, Metallorum item et reliquorum Mineralium naturae, vires, atque usus exquisitissime explicantur ... Venetiis apud Juntas 1553.

S. 290 v. Conradi Gesneri Excerptorum et Observationum de Thermis liber. 
Monographien. Mit Geßner waren noch etwa dreißig andere Zeitgenossen Mitarbeiter an diesem Werk, meist natürlich Italiener. Der Beitrag Geßners handelte «De Thermis et Fontibus medicatis Helvetiae et Germaniae». Er spricht darin zuerst von den Thermen im Allgemeinen und der Herkunft des Wortes und erwähnt dabei die Thermopylen. Dann schildert er die römischen Bäder mit ihrem Trepidarium, Auskleideraum, dem Sudatorium oder Laconicum oder Calidorium, Hypocaustum oder Pyriaterium, d. h. Schwitzbad, dem Solium oder Labrum calidum embasis, dem Warmwasserbad, dem frigidum Solium oder Kaltwasserbad und schließlich dem Abreiberaum, in dem sich die Badenden auch wieder anzogen, meist Apodypterium genannt. Alle diese Teile bildeten bei den Römern ein Balneum, während die Deutschen jeden Teil davon ein Bad nennen. Geßner erklärt, er selber unterscheide eine Therme und ein Bad nicht anders von einander als eine Species und ein Genus. In einem zweiten Kapitel behandelt er kurz Herkunft und Ursachen der Thermen und bezieht sich dabei auf Georg Agricola (1490-1555), Stadtarzt und Bürgermeister von Chemnitz und Begründer der Mineralogie, der 1546 in Basel ein Werk veröffentlicht hatte "De ortu et causis subterraneorum». ${ }^{31}$ Agricola war mit Geßner Mitarbeiter am «Corpus Venetum» mit einem Beitrag «De natura eorum quae ex terra effluunt». Als weitern Gewährsmann zitiert Geßner Hieronymus Cardanus (1501-1576), den berühmten italienischen Mathematiker, Arzt und Philosophen und sein aus einundzwanzig Büchern bestehendes Werk «De subtilitate» und zwar das zweite Buch. ${ }^{32}$ Auch von Cardanus enthält das «Opus Venetum» eine Arbeit, «An balneum articulari morbo competat». Das große Werk «De subtilitate» ist 1550 in Nürnberg zum ersten Mal im Druck erschienen. Man ist immer wieder erstaunt, wie sehr Geßner die zeitgenössische Literatur kannte und die neuesten Forschungen verfolgte, auch auf Gebieten, die ihm nicht besonders nahe lagen.

Nach dieser Einleitung kommt er auf die schweizerischen Heilquellen zu sprechen, aus eigener Anschauung wie aus Literaturkenntnis: «Conradi Gesneri excerptorum et observationum de Thermis liber primus». Er beginnt mit Baden, dem damals weitaus berühmtesten Bad der Eidgenossenschaft und zwar mit einem historischen Exkurs. Er erwähnt, was Tacitus über dieses Bad geschrieben hat, kennt die ausgegrabenen Monu-

31 G. Agricola, vgl. Allg. D. Biogr., Bd. 1, S. 143 ff., sowie neuere Spezialliteratur.

32 H. Cardanus, vgl. «Lexikon d. hervorr. Ärzte», Bd. 1, S. 829 und Ergänzungsbd. S. 158 mit Lit.-Angaben. 
mente aus der Zeit Vespasians, gibt den seither oft abgedruckten Brief des italienischen Humanisten Poggio aus dem beginnenden 15. Jahrhundert wieder, der in amüsanter Weise seine damaligen Badeerlebnisse schildert, zitiert die Arbeiten der oben genannten Gundelfingen und Alexander Sytz und bespricht̃ schließlich die Bäder selber, deren Wasser reich an Schwefel sei und etwas Alaun enthalte. Dann folgt die Beschreibung von Pfäffers. Er kennt die Badeschrift von Paracelsus, wie auch die Erzählungen des Zürcher Chorherrn Felix Hämmerlin in seinem «De balneis naturalibus liber». Geßner hat dieses Bad zweimal besucht, selber aber offenbar nicht gebraucht, schreibt er doch: «Per biduum uti volui». Die Angabe, die Quelle enthalte Gold, Schwefel und Salpeter, entnahm er wohl andern Schriftstellern. Im Bad Fideris im Prätigau imponierte ihm vor allem das Pumpwerk; ein Bild davon gab er seiner Arbeit bei. Er glaubte, daß diese Quelle viele metallische Bestandteile oder Heilstoffe aus dem Erdinnern enthalte. Offenbar hat er sie selber ausprobiert, berichtet er doch: «Hoc balneum etiam ipse congressus sum experimenti causa». Die Quellen bei Brig kannte er aus der Schilderung von Sebastian Münster, die Bäder von Leuk aus eigener Anschauung und Erfahrung, da er dort eine zwanzigtägige Badekur gemacht hatte. Ihre Wasser hielt er für kupferhaltig: «Odorem, saporem caloremque a cupro trahunt».

Die Zürcher Bäder erfuhren trotz ihres bescheidenen Wertes eine besonders liebevolle Darstellung. Das heute eingegangene Bad von Urdorf nennt er «splendide constructa». Es wurde angeblich aus drei Quellen gespeist. Der Angabe, die eine enthalte Schwefel, die andere Alaun und die dritte Kupfer, mißtraute er, sagt er doch: «Siquid talem continent, perexiguum est». Offenbar hat er dieses Wasser selber untersucht. Das Wengibad bei Aeugst hielt er für ton- und leicht gipshaltig; seine Frau soll es einmal wegen eines alten Krampfaderleidens mit ziemlichem Erfolg gebraucht haben. Geßner erwähnt auch die zwei kleinen, längst vergessenen Bäder von Grüningen und Hinteregg am Pfannenstiel, welch letzteres schon zu seiner Zeit nicht mehr im Gebrauch war. Auch das Nidelbad bei Rüschlikon schildert er aus eigener Anschauung, dessen Wasser für schwefelhaltig galt. Es scheint, daß alle diese Bäder schon vor Geßner untersucht und wohl auch beschrieben worden sind; manches ist offenbar im Lauf der Jahrhunderte verloren gegangen. Geßner konstatierte einen fauligen Schwefelgeruch, wollte daraus aber nicht auf die Anwesenheit von Schwefel schließen, da er diesem Geruch immer begegne bei Quellen. 
die in Sumpfgebiet entspringen, zumal in Zisternen. Der Boden, über den sie fließe, werde durch sie gerötet.

Es folgen kurze Erwähnungen der Quellen von Lustdorf im Kanton Solothurn, Nuolen am Zürichsee, Rietbad im Toggenburg, Alvaneu im Bündnerland, deren Schwefelgehalt ihm bekannt ist, Schamps, offenbar die heute in Andeer benutzte Quelle, Osterfingen im Kanton Schaffhausen, schließlich einer kleinen Quelle, eine Meile von Basel entfernt und des Wassers von Knutwil im Kanton Luzern.

Die deutschen Quellen ${ }^{33}$ kannte Geßner kaum aus eigener Anschauung; er war auf die Mitteilungen anderer Schriftsteller angewiesen, auf die Angaben eines Leonhard Fuchs in Tübingen, Gundelfingens in Freiburg i. Br. u. a. Er führte sie in alphabetischer Reihenfolge auf, einige offenbar mehr als einmal, je nach den Berichten seiner Gewährsmänner. Die süddeutschen Bäder nehmen einen besonders großen Raum ein. Er nennt die Bäder Antogast bei Oppenau; Aachen; Abbach ob Regensburg; Baden bei Wien; Baden-Baden; Budapest; Eger in Böhmen; Elenbogen in Böhmen, (das heutige Karlsbad); Ems bei Koblenz, Ems bei Konstanz (unbekannt), vermutlich Hohenems; Gebersweiler, Gebweiler i. E., als Bad ebenfalls unbekannt: Göppingen, auch seinen Sauerbrunnen; Gastein; Rothenburg a. d. Tauber; Überlingen am Bodensee, eine heute nicht mehr benutzte Quelle; Wildbad im Schwarzwald; Zell bei Calw im Schwarzwald, unbekannte Quelle; Wiesbaden. Es folgt dann eine längere Aufzählung von Badeorten, welche G. Agrippa in seinem vorgenannten Werk bekannt gibt und zum Schluß ein Lobgedicht von Joachim Camerarius (1500-1574) auf das Vogesenbad Plombières.

Schon im Jahr 1565 gab ein anderer deutscher Mediziner, Winter von Andernach (1487-1574), ${ }^{34}$ besonders in Paris und Straßburg tätig, eine viel umfangreichere Bäderliste heraus. Sein in Straßburg herausgekommener «Commentarius de balneis et aquis medicatis in tres dialogos distinctus» zählt bereits fünfundsiebzig Bäder auf. 1568 erschien in Dillingen bei Mayen von Martin Ruland (1532-1602) ${ }^{35}$ eine Schrift «Hydratice seu Aquarum medicarum sectiones quatuor», welche in einer ersten Abteilung die verschiedenartigen Bäder mit den speziellen Krankheitsindikationen aufzählte, um im folgenden Teil siebenundfünfzig deutsche Bäder mit

33 C. Gesneri de Thermis Germaniae ordine literarum liber secundus, S. 296 v.

34 Winter von Andernach, vgl. «Lexikon d. hervorr. Ärzte», Bd. 2, S. 883 f. und die einschlägigen medizinhist. Handbücher. Geßner schätzte ihn nicht.

35 M. Ruland, vgl. «Lexikon d. hervorr. Ärzte» Bd. 4, S. 922 mit Lit.-Angaben. 
ihrer qualitativen Zusammensetzung mitzuteilen. Ruland nimmt in seinem Werk wohl Bezug auf Winter v. Andernach, nicht aber auf Geßner. Im Exemplar der Zürcher Zentralbibliothek findet sich auf der letzten Seite ein interessanter handschriftlicher Vermerk aus dem 16. Jahrhundert. Verzeichnus deren Beder, so bey uns gemain und breuchig sind», aus der Feder eines Arztes der Nordschweiz oder Süddeutschlands. Er zählt folgende Bäder auf: Baden, Cellerbad bei Calw, Embs ob Costantz, Fliehen (Flüh) bei Basel, Leuck im Wallis, Löffingen (gemeint ist Bad Boll), Neuhad bei Villingen, Pfäffers, Wildbad bei Pforzheim, Überlingerbad, Margrafenbad beim stettlin Baden (Baden-Baden). Diese Aufzählung ist deshalb interessant, weil sich daraus ergibt, daß Geßners Aufzählung wohI doch weniger zufällig ist, als dies auf den ersten Blick erscheinen möchte. Ruland selbst erwähnt auch das Bad Elbogen, setzt aber Carolinae voran, sodaß nicht daran zu zweifeln ist, daß es sich um Karlsbad handelt. Auch er erwähnt Fliehen (Flüh im Kanton Solothurn).

Später war Geßner mit dieser Darstellung nicht mehr recht zufrieden, und noch in den letzten Lebensjahren beschäftigte ihn der Plan einer Neubearbeitung, welcher wie viele andere Pläne des fleißigen Mannes wegen seines frühzeitigen Todes nicht zur Ausführung kam. So schrieb er 1561 an seinen englischen Freund John Caius (1510-1573), kgl. Leibarzt, der sich mit dem Plan trug ein Buch über die englischen Bäder zu schreiben, er solle die Ausgabe nicht überstürzen, ${ }^{36}$ und seinem Freund Achilles Gasser berichtete er 1562: «Ich bereite eine neue Ausgabe meines Buches über die Heilquellen Deutschlands vor. Ich würde mich freuen, wenn Du etwas zu seiner Ausstattung beitragen könntest, wie wenig es auch wäre. ${ }^{37}$ Ständig sammelte er neues Material, und offenbar arbeitete er fleißig an der Verbesserung der Methoden zur qualitativen Beurteilung der Mineralwasser. Um sich über den therapeutischen Wert der einzelnen Quellen ein genaueres Bild machen zu können, machte er viele eigene Versuche. Daß Geßner schon vor der Herausgabe seines Bäderbuches zwanzig Tage im Leukerbad zugebracht hatte, wurde bereits erwähnt. In Baden scheint er 1547 eine erste Kur gemacht zu haben, die er aber in der Folge oft wiederholte, so 1560, 1562, 1563 und 1564. Dabei gestaltete

36 John Caius, vgl. Dictionary of national biography, Bd. 3, S. 673 ff. und Geßners Brief an Caius vom 29. Aug. $1561 \mathrm{im}$ 3. Buch Epist. medicinalium, S. 134 ff., hgg. von C. Wolf in Zürich, 1577.

37 Epist. medicin. Lib. 1, pg. 23. Achilles Pirmin Gasser, (1505-1577), Stadtarzt in Feldkirch und später in Augsburg. Allg. D. Biogr., Bd. 8, S. 396 f. 
er die Kur völlig anders, als dies heute gebräuchlich ist. Zunächst einmal verband er auch in Baden die Badekur mit einer Trinkkur, zur Stärkung des Magens. Er begann am ersten Tag mit fünfzig Unzen oder 11/2 Liter und bemerkte stärkere Schweißabsonderung, stieg am folgenden Tag auf 75 Unzen oder 2 Liter, wobei sich vermehrter Urinabgang einstellte, am dritten Tag auf 100 Unzen, wonach Durchfälle auftraten. ${ }^{38}$ In der Regel machte er diese Trinkkur vier Tage in aufsteigenden Mengen, um in den folgenden Tagen die Trinkmenge in umgekehrter Folge wieder zu vermindern, sodaß eine Kur acht bis neun Tage dauerte. Er badete morgens und abends, bis eine Stunde lang und verband die Bäder mit Übergießungen. Im vorerwähnten Brief an Achilles Gasser schrieb er: «Ich war neulich vier Tage in Baden, um meine Frau, die dort badete, zu besuchen. In diesen vier Tagen habe ich mir im Bad einen großen Schwamm auf den Kopf gelegt und eine Stunde oder noch länger morgens und abends warmes Wasser aus der Quelle darüber gießen lassen. Ich habe gefunden, daß dieses Verfahren besser ist für mich, als ein wiederholter Guß von oben aus einem kleinen Gefäß, wie ich es vor zwei Jahren versucht habe. ${ }^{39} 1560$ verbrachte er mehrere Wochen in Baden, weil er von einer schweren Ischiasaffektion geplagt war, welche jeder medikamentösen Behandlung getrotzt hatte. ${ }^{40}$ Nach einer solchen Trinkkur hatte er zu Hause zwei bis drei Wochen lang täglich schleimig-flüssige Stühle, die er dann mit Mehlmus stopfte. Dabei befand er sich aber sehr wohl und hatte weit weniger Magenbeschwerden als zuvor. 1564 schrieb er an Crato von Crafftheim, den kaiserlichen Leibarzt (1519-1585), einen Einfluß auf die Darmtätigkeit verspüre er nicht wie die meisten andern Patienten; zu diesem Zweck müsse er ein Medikament einnehmen. Dagegen purgiere das Wasser die Blase, bringe ihn zum Schwitzen und hebe seinen Appetit. Mit dem Urin entleerten sich jeweils Schleim, Blutgerinnsel und feiner Sand. ${ }^{41}$ Bei andern Patienten hatte er mit einer solchen steigenden Trinkkur Erfolg bei Blasenbrennen. Diese Art der Trinkkuren hatte er italienischen Schriften entnommen. Dem Sohn Bullingers (1504-1575), der an

38 Gsell-Fels, Bäder und Kurorte d. Schweiz, 2. Aufl., Zürich, 1890, S. 504.

39 Epist. medicin. lib. I, pg. 23.

40 Epist. medicin. lib. III, pg. 88, an Taddaeus Dunus, Aug. 1560. Über T. Dunus vgl. Salzmann, Taddeo Duno von Locarno als Stadtarzt in Zürich, Vierteljahrsschr. d. natf. Ges. i. Zürich, 1940, 3. und 4. H., S. 337-344.

41 Epist. medicin. lib. I. pg. 18, Brief an Crato v. Krafftheim (1519-1585), Leibarzt von Ferdinand I., Maximilian II. und Rudolf II. Vgl. «Lexikon d. hervorr. Ärzte», Bd. 2, S. 138 f. 
Schwindsucht und Blutspeien litt, gab er den Rat, er dürfe nur von der Quelle beim Ochsen trinken, weil diese nur etwas Alaun, aber keinen Schwefel enthalte, worin sich Geßner aber sicher geirrt hat, sofern es sich bei jenem Brunnen nicht um ganz gewöhnliches Brunnenwasser handelte, da die Zusammensetzung aller Badenerquellen so ziemlich die gleiche ist. ${ }^{42} 1561$ unternahm Geßner eine mehrwöchige Exkursion ins Bündnerland, zusammen mit seinem Freund Fabricius Montanus in Chur (1527-1566) und Johannes Bauhin, einmal, um zu botanisieren, dann aber auch, um Bäder und Quellen zu untersuchen. ${ }^{43}$ Dabei kam er zum ersten Mal ins Unterengadin, nach Schuls und Vulpera. Ein Jahr darauf schrieb er an Gasser, seit er von jener salzhaltigen Quelle, einem Wunder der Natur, getrunken habe, fühle er sich immer wohl, wohler als vor mehreren Jahren. Das Wasser dieser Quelle hatte er eingedampft, um den Rückstand zu untersuchen; er glaubte, darin reichlich Salpeter gefunden zu haben. Er schrieb darüber an Caius: "Inter alias fontes (de quibus scribere non vacat) unum reperi, cuius aqua decoctione consumpta nitrum verum (ut mihi quidem videtur) syncerumque relinquit copiosum». Auch ihm berichtet er, er habe diese Reise unternommen «ut fontium naturas addiscerem». ${ }^{44} \mathrm{Im}$ folgenden Jahr schickte Fabricius Wasser von einer Quelle bei Chur, mit Schlamm vermischt, einer Quelle, welche Geßner offenbar mit ihm schon im vorigen Jahr besucht hatte. ${ }^{45}$ Geßner bedankt sich sehr dafür und will das Untersuchungsergebnis mit dem letztjährigen vergleichen.

Zum Schluß seien die Anweisungen zur Untersuchung von Mineralquellen wiedergegeben, die Geßner in seinen «köstlichen Arzneischatz» aufgenommen hat und welche von Falloppio stammen:

Man läßt sich einen gemauerten, lehmverputzten Brennofen bauen, mit Feuerungsloch und Rauchabzug, der über der Feuerung ein leeres Fach enthält. In dieses Fach stellt man ein breites, mit Sand gefülltes, irdenes Gefäß. Nun füllt man ein gewöhnliches Uringlas oder ein Kolbenglas mit Urin und stellt es so in dieses Gefäß, daß es halb im Sand steckt. Auf die Öffnung des Uringlases kommt ein Helm, den man gut abdichtet, daß

42 Epist. medicin. lib. III., pg. 106, an Theodor Zwinger, (1533-1588), Med. Prof. in Basel. Vgl. Wolf, 1. c., Bd. 3, S. 119 f. und Burckhardt, 1. c. S. 89-95.

43 Über Johann Schmid oder Fabricius Montanus, Pfarrer in Chur und Botaniker, vgl. H. Fischer, J. Fabricius Montanus und Conrad Geßner, Vierteljahrsschr. d. natf. Ges. i. Zürich, 1940, 3. und 4. H., S. 322 ff.

44 Epist. medicin. lib. III., pg. 134.

45 Epist. medicin. lib. III., pg. 92 v. 
kein Dampf entweichen kann. In diesem Helm befestigt man eine nach unten laufende gläserne Röhre, die an ihrem untern Ende durch ein mit kaltem Wasser gefülltes Gefäß geführt wird. Glasrohr und Helm werden wieder gut abgedichtet durch Umwickeln mit einer mit Kleister bestrichenen leinenen Binde. Man kann das Wasser auch im Balneum Mariae verdampfen, doch ist dieses Verfahren einfacher.

Wird nun ein Feuer angezündet, erwärmt sich der Sand und nachher auch das Wasser, das bald zu verdampfen beginnt. Der Dampf sammelt sich im Helm, kondensiert sich und fließt durch das Glasrohr ab und bleibt flüssig, da dieses abgekühlt ist. Der Verdampfungsrückstand, die Hefe, wird dann untersucht. "Auff solche weise kanstu erfahren was für Geister mit dem Wasser vermischet seyen", wie es in der deutschen Ausgabe des «köstlichen Arzneischatzes» heißt.

Der Rückstand wird nun herausgenommen und an der Sonne getrocknet. Noch bevor man ihn herausnimmt, gleich nach Beendigung der Destillation, soll man aber an der Hefe riechen, um festzustellen, ob sie Thon oder andere Erden enthält, da man dies anders nicht feststellen kann. Enthält sie «Rötel» (Rubrica) oder «Berckgelb»(Ochra), erkennt man das ohne weiteres am Geruch.

Ist die Hefe erkaltet, verreibt man einen Teil zwischen den Fingern; Reuschelgelb (Sandaraca), d. i. Arsensulfür, Schwefel und Auripigment lassen sich dabei voneinander unterscheiden.

Der Rest des Rückstandes wird nun, auf einem Brett ausgebreitet, an der Sonne getrocknet. Kleine Alaunpartikel sind dabei im Sonnenlicht als solche erkennbar. Auch Salze, Schwefel, Salpeter werden so sichtbar gemacht, nicht aber Metalle wie Gold, Silber, Eisen, Zinn und dergleichen.

Die so getrocknete und untersuchte Hefe wird nun auf ein Eisenblech gelegt, das zum Glühen gebracht wird. Kalk und Kiesel verbrennen dabei nicht und werden nur etwas heller. Wird eine Substanz, auch nicht verbrennend, sofort hell weiß, ist es Gips, heller weiß und rascher als Kalk und Marmel. Alle drei aber verbrennen nicht. Verbrennender Schwefel wird sofort am Geruch erkannt.

Salz und Salpeter verbrennen beide mit starkem Leuchten (scintillabunt), wobei Salz beim Verbrennen erst noch knistert, im Gegensatz zu Salpeter.

Bleinachweis ist Falloppio nie gelungen, doch meint er, es würde bei dieser Prozedur wohl schmelzen. Bei Vorhandensein von Bleiweiß wird die Hefe mennigrot, da Mennige aus Bleiweiß gemacht wird. 
Metalle können auf diese Weise nicht eruiert werden. Man kann sie nicht anders feststellen, als durch Zerstören der Hefe, wobei sich jedes Metall verwandelt und in sein Exkrement übergeführt wird, als welches es dann erkannt werden kann. Die Hefe wird zu diesem Zweck mit Essig oder Ätzwasser versetzt; den eingetrockneten Rückstand kann man auf metallische Exkremente untersuchen. Findet man Rost darauf, ist das ein Zeichen für Vorhandensein von Eisen, Grünspan ein solches von Kupfer. Man darf aber nicht jede grüne Farbe für Grünspan ansprechen; sie kann auch von grünem Bolus oder Rost herrühren. Wird solche grüne Hefe in destillierten Essig gelegt, wird Bolus noch grüner, Rost aber schwarz.

Die Galläpfelprobe kennt Falloppio, wenn auch nicht ganz zutreffend, da er auch Alaun damit nachweisen will.

Auf Geruch und Geschmack soll man sich nicht verlassen, da es sich meist um Gemische handelt, die ein sicheres Urteil nicht zulassen.

Mag auch so ziemlich alles in dieser Untersuchungsanweisung heute überholt sein, handelt es sich doch um einen ersten, hochwichtigen Anfang qualitativer Untersuchung von Mineralwasser, der erst lange nachher überholt wurde. Wieviele solche Untersuchungen Geßner noch selber durchführen konnte, weiß man nicht. Da er selber sehr fleißig destillierte, schon aus pharmazeutischen Gründen, wie man aus seinen Briefen weiß, ist es durchaus möglich, daß er umfangreichere Untersuchungen gemacht hat; auf keinen Fall hat er sich nur literarisch dafür interessiert. Eine zweite Ausgabe seines Bäderbuches hätte wahrscheinlich allerlei Zusätze gebracht. Man wird aber schon jetzt nicht bestreiten können, daß Geßner mit Recht auch der Name eines Vaters der schweizerischen Balneologie gebührt.

Bernhard Milt.

\section{Descartes und Theophrast von Eresos}

In seiner berühmten, zuerst anonym im Jahre 1637 erschieneners Schrift: «Discours de la méthode pour bien conduire sa raison et chercher la vérité dans les sciences» hat Descartes der Physik die sicheren mathematischen Grundlagen geschaffen. Im ersten Teil seiner Abhandlung legt er die Gründe dar, welche ihn veranlaßt haben, eine neue Methode wis. 